\title{
Construction of Lentivirus-Based Reference Material for RT-PCR Detection of Middle East Respiratory Syndrome Coronavirus and Its Application in External Quality Assessment
}

\author{
Donggen Zhou*, Jie Luo \\ Ningbo International Travel Healthcare Center, Ningbo, China \\ Email: *zhoudg@nbciq.gov.cn
}

How to cite this paper: Zhou, D.G. and Luo, J. (2018) Construction of Lentivirus-Based Reference Material for RT-PCR Detection of Middle East Respiratory Syndrome Coronavirus and Its Application in External Quality Assessment. Advances in Microbiology, 8, 506-518.

https://doi.org/10.4236/aim.2018.87035

Received: June 25, 2018

Accepted: July 24, 2018

Published: July 27, 2018

Copyright $\odot 2018$ by authors and Scientific Research Publishing Inc. This work is licensed under the Creative Commons Attribution International License (CC BY 4.0).

http://creativecommons.org/licenses/by/4.0/

\begin{abstract}
Nucleic acid amplification technologies (NAT) have been used most for rapid detection of Middle East Respiratory Syndrome coronavirus (MERS-CoV) since MERS-CoV was first found in 2012. It is important to develop stable and safe reference materials for assessing the quality of NAT kits and external quality assessment (EQA) of different labs. In this study, the MERS-CoV RNA fragments including $u p E, O R F 1 b$ and $N$ were packed within human immunodeficiency virus type 1 (HIV-1) like particles. The lyophilized virus-like particles (VLPs) were found stable at $37^{\circ} \mathrm{C}$ or below and safe to be used not only as the control material for PCR detection of MERS-CoV, but also as the reference material for EQA. In an EQA organized by Ningbo International Travel Healthcare Center in China, 49 participating institutes achieved 100\% agreement for detecting MERS-CoV using various commercial diagnosis kits and different extraction methods. But the different $\mathrm{Ct}$ values reported by the different labs for the same sample implied that there needed to standardize the RNA extraction method and/or the PCR detection conditions between the labs.
\end{abstract}

\section{Keywords}

MERS-CoV

\section{Introduction}

Since the Middle East Respiratory Syndrome coronavirus (MERS-CoV) was first found on 22 September 2012 [1], it has caused a few outbreaks. In the latest out- 
break which happened in 2015, 186 South Koreans were infected and 38 of them died. Owing to a lack of effective intervention and treatment, early diagnosis and isolation are the primary methods to control MERS-CoV infection [2]. For early detection of MERS-CoV [3], real-time reverse transcription polymerase chain reaction (RT-PCR) targeting $u p E, O R F 1 a$ or $O R F 1 b$ fragments of MERS-CoV is the method used most commonly and frequently [4] [5].

Most of these kits utilize plasmids or in vitro-transcribed RNA as the positive controls for the assays. However, these materials could not offer control for the extraction procedure. Recombinant bacteriophages such as MS2 could be used to control the efficiency of the extraction procedure [6] [7] [8] and the presence of PCR inhibitors in RT-PCR assays. Since the maximum load volume of MS2 for foreign gene is about $2 \mathrm{k}$, it might need to construct a few recombinant bacteriophages to accommodate different RNA targets of MERS-CoV. Wild type virus preparations could be used as controls also [9] [10], but these are limited to those laboratories which have access to biosafety level 3 facilities [11]. Furthermore, inactivation of high titre virus stock by a single method does not assure safety, and most published procedures that produce inactive viruses through a combination of methods, might result in disruption of viral RNA making its suitability as NAT standard uncertain [12].

Because MERS-CoV exists in the natural reservoir of camels, it is believed that MERS-CoV infection would come back sooner or later. China is one of the countries in closest contact with Middle East, and a large number of people entered and exited China, especially in harbor cities. As a result, there was a risk that MERS-CoV would enter China again. Screening of high-risk populations from MERS-CoV affected regions was therefore essential not only for control and prevention of MERS-CoV in Chinese native, but also being part of the global task of fighting the infection. The availability of reference materials for the comparison of the sensitivity of different assays, for the validation of recently developed point of care technologies and for the harmonization of inter- and intra-laboratory results is therefore fundamental for the MERS-CoV control.

In this study, we packed the MERS-CoV RNA fragments into human immunodeficiency virus type 1 (HIV-1) like particles using the lentivirus package system. The pellets produced finally, are safe, non-replicating, freeze-fried specimen that can function as the control materials for the entire diagnostic procedures from virus RNA extraction to nucleic acid amplification. An external quality assessment (EQA) based on the virus-like MERS-CoV organized by Ningbo international travel healthcare center to cover 49 labs in China showed that the virus-like particles (VLPs) are also suitable to be used as the reference materials for preparing the sample panel in EQA.

\section{Materials and Methods}

\subsection{Plasmids Preparation}

The nucleotide sequences of MERS-CoV upE (27,180 bp - 27,514 bp), ORF1b 
$(17,700 \mathrm{bp}-19,000 \mathrm{bp})$ and Nseq $(28,500 \mathrm{bp}-30,000 \mathrm{bp})$ are from NCBI (GenBank accession number KT029139.1) [13]. Fragment $1 b N$ containing ORF1b and Nseq were synthesized by GenScript Company (Nanjing, China). Fragment upE was provided by Professor ZhengliShi (Wuhan Institute of Virology, Chinese Academy of Sciences, China). These two fragments were sub-cloned into the pCMV-MCS-CopGFP vector (clone sites and primers used are listed in Table 1), forming the expression plasmids pCMV-MCS-upE and pCMV-MCS-1bN. Lentiviral vector $\mathrm{pCMV-MCS-CopGFP} \mathrm{is} \mathrm{a} \mathrm{commercial} \mathrm{lentiviral} \mathrm{vector} \mathrm{in} \mathrm{which} \mathrm{the}$ Cytomegalovirus (CMV) major immediate early promoter (MIEP) is at the upstream of the multi cloning sites and the reported gene green fluorescence protein (GFP). The constructed plasmids were confirmed by sequencing (Tsingke Co., Wuhan, China) and analyzed by software DNAstarMegAlign.

\subsection{Express and Purify MERS-CoV VLPs}

The MERS-CoV VLPs were produced by the third generation lentivirus package system, which contains four plasmids: pCMV-MCS-CopGFP, PLP1, PLP2 and VSVG. To block the expression of MERS-CoV proteins, start codons were removed from every fragment. To increase the biosafety of the system, the long terminal repeats in the lentiviral vectors are defective $(\Delta \mathrm{U} 3)$, an internal promoter is missing and no envelope protein is expressed in the transfected cells, rendering the HIV-like particles non-infectious. The lentiviral particles were generated by transfection of $5 \times 10^{6}$ HEK293T-17 (ATCCCRL-11,268) cells in a $10 \mathrm{~cm}$ dish with a mixture of $18 \mu \mathrm{L}$ of lipofectamine 3000 reagent (Promega), $12 \mu \mathrm{g}$ of PLP1, $6 \mu \mathrm{g}$ of PLP2, $3 \mu \mathrm{g}$ of VSVG and $9 \mu \mathrm{g}$ of pCMV-MCS-upE or pCMV-MCS-1bN in $650 \mu \mathrm{L}$ Dulbecco modified essential media (DMEM, Gibco). After incubation for $5 \mathrm{~min}$ at room temperature, the mixture was added drop-wise to $8 \mathrm{~mL}$ of DMEM supplemented with $10 \%$ fetal calf serum. Then the cells were cultivated at $37^{\circ} \mathrm{C}$ with $5 \% \mathrm{CO}_{2}$ for 72 hours. The supernatant was harvested and filtered using a $0.45 \mu \mathrm{M}$ filter (Millipore). The VLPs in the supernatant were purified by ultracentrifugation at $201,400 \mathrm{~g}$ at $4^{\circ} \mathrm{C}$ for $2 \mathrm{~h}$ on a $20 \%$ sucrose cushion in 50 $\mathrm{mM}$ sodium phosphate buffer using a SW70 rotor in a Beckman Optima LE-80K Ultracentrifuge. Then the pellets were washed once with phosphate buffer saline (PH7.4) and harvested by ultracentrifugation at 201,400 $\mathrm{g}$ at $4^{\circ} \mathrm{C}$ for 1 hour. Finally, the MERS VLPs were re-suspended in $100 \mu \mathrm{L}$ PBS (PH7.4) with

Table 1. Primers used for construction of MERS-CoV virus-like particles. Restriction sites are underlined. GAATTC: EcoRI; GGATCC: BamHI.

\begin{tabular}{ccc}
\hline Name & Sequence (5'-3') & Tm \\
\hline MERSVLP-upE-F & CGAATTCCTACATTCCACTGTTT & 61 \\
MERSVLP-upE-R & CGTGGATCCCGTTAAACCCACTCGTCAG & 72 \\
MERSVLP-1bN-F & CGGAATTCTTTTATTACTGCCAATCC & 63 \\
MERSVLP-1bN-R & TCGGATCCAGGTGACAGTCTTTAACAT & 67 \\
\hline
\end{tabular}


5\% trehalose dehydrate (Sigma) and 1\% Bovine Serum Albumin (BSA) at concentration of about $10^{8}-10^{9}$ copies/mL and freeze-dried.

\subsection{Freeze-Drying Procedure}

In separate procedures, the final preparations were aseptically dispensed in 0.1 $\mathrm{mL}$ aliquots into $2 \mathrm{~mL}$ glass vials. The mixture was pre-frozen at $-80^{\circ} \mathrm{C}$ for 12 hours, following by lyophilized at $-56^{\circ} \mathrm{C}$ for 6 hours in a freeze-dryer (scanvacoolsave ${ }^{\mathrm{TM}} 55-9$, Germany).

\subsection{Transmission Electron Microscopy}

An aliquot of $20 \mu \mathrm{L}$ MERS-CoV VLPs solution in the supernatant after the sucrose-purification were soaked with a Formvar carbon-coated copper grid for 10 minutes. Then the grids were removed and washed 3 times using purewater. After that, the grids were stained with $20 \mu \mathrm{L}$ of $2 \%$ phosphotungstic acid (PTA, PH 6.8) for $30 \mathrm{~s}$ and air-dried. Finally, Transmission Electron Microscopy was performed (H-7000FA, Hitachi).

\subsection{Assessment of MERS VLPs}

The actual copies of RNA in the freeze-dried MERS VLPs powder was quantified using droplet digital PCR (ddPCR). The ddPCR was conducted on a QX200 ${ }^{\mathrm{Tm}}$ ddPCR system (Bio-Rad) according to the manufacturer's recommendations. Briefly, the lyophilized powder was reconstituted with $140 \mu \mathrm{L}$ water. Then RNA was extracted from the solution using a QIAamp viral RNA minikit (Qiagen). The RNA obtained was collected with $60 \mu \mathrm{L}$ water. After that, $5 \mu \mathrm{L}$ of RNA solution was reverse-transcripted in cDNA using the GoScript ${ }^{\mathrm{TM}}$ Reverse Transcription system (Promega, USA) with specific primers in accordance with the manufacturer's instructions. Finally, PCR reaction mixtures were prepared by mixing $11 \mu \mathrm{L}$ of $2 \times$ Supermix, $0.25 \mu \mathrm{M}$ of each of the probes and $0.9 \mu \mathrm{M}$ of each primers, $2 \mu \mathrm{L}$ of the cDNA solution and water with a total volume of $22 \mu \mathrm{L}$. The primers and probes were bought from Liferiver (Shanghai, China). Droplets were generated using an Automate Droplet Generator (Bio-Rad) where a vacuum was applied to the outlet wells to simultaneously partition the PCR mixtures into nanoliter sized droplets. The PCR plate obtained was subsequently heat-sealed with pierceable foil using a PX1 ${ }^{\mathrm{rm}} \mathrm{PCR}$ plate sealer (Bio-Rad) and then amplified in a conventional thermal cycler (C100 Touch, Bio-Rad). The thermo-cycling parameter was set as: de-naturation $95^{\circ} \mathrm{C}$ for $10 \mathrm{~min}$, then 40 cycles of denaturation at $94^{\circ} \mathrm{C}$ for $30 \mathrm{~s}$, annealing at $60^{\circ} \mathrm{C}$ for $1 \mathrm{~min}$ (temperature ramp $2^{\circ} \mathrm{C} / \mathrm{s}$ ) and, finally, incubation at $98^{\circ} \mathrm{C}$ for $10 \mathrm{~min}$. After the cycling, the 96 -well plate was fixed into a plate holder and placed in a Q200 Droplet Reader (Bio-Rad). Finally the data obtained were analyzed by the analysis software package provided (QuantaSoft, Bio-Rad). Droplet counts below 10,000 droplets were unacceptable and discarded.

The stability of the lyophilized VLPs powder was tested by storing the powder 
under $4^{\circ} \mathrm{C}$, room temperature and $37^{\circ} \mathrm{C}$ for one week. Then the copies of RNA in the freeze-dried MERSVLPs powder were checked again using the above procedure.

\subsection{Sample Panel Preparation and External Quality Assessment (EQA)}

A sample panel consisting of three negative and seven positive samplesranging from $8 \times 10^{2}$ to $5 \times 10^{7}$ copies $/ \mathrm{mL}$ of the VLPs was designed to use in an external quality assessment (EQA) organized by Ningbo international travel healthcare center. Briefly, one bottle of the lyophilized powder was reconstituted using 1 $\mathrm{mL}$ of a virus preservation solution (DMED with $0.1 \%$ BSA, $10 \%$ glycerol and antibiotics). Then the solution was diluted in series 10 fold from $10^{2}$ to $10^{7}$ copies/mL. A $140 \mu \mathrm{L}$ volume of each sample was extracted using QIAamp viral RNAmini kit (Qiagen, US) following the manufacturer's instructions, and elutedin $60 \mu \mathrm{L}$ water. A commercial PCR kit (Liferiver, Shanghai, China) was used to test the solutions. At the same time, 100 aliquots of $40 \mu \mathrm{L}$ were taken from the solutions with three different concentrations of the MERSVLPs (Sample SP, $1 \times$ $10^{6}$ copies $/ \mathrm{mL}$; Sample NP, $5 \times 10^{5}$ copies/mLand Sample LP, $1 \times 10^{5}$ copies $/ \mathrm{mL}$ ). A homogeneity validation test was performed by randomly selecting 10 aliquots for the RT-PCR test. To evaluate the stability of the reconstituted particles, another 4 aliquots of $40 \mu \mathrm{L}$ were taken from the solutions of the sample SP, NP and $\mathrm{LP}$, and store at $-70^{\circ} \mathrm{C},-20^{\circ} \mathrm{C}, 4^{\circ} \mathrm{C}$ and $37^{\circ} \mathrm{C}$ for 7 days, respectively. The RT-PCR test was performed at $1 / 3 / 7$ days to check the stability during the storages.

The EQA was attended by 49 laboratories mainly under Chinese Entry-exit inspection and quarantine Bureau and the Centers for Disease Control and Prevention of China. A sample panel consisting different concentrations of the MERS-CoV VLPs was prepared and transported to the laboratories with ice box. The test results were collected and evaluated within one month.

\section{Results}

\subsection{Generation of MERS-CoV Virus like Particles}

Two constructed lentiviral vectors, pCMV-MCS-1bN containing MERS-CoV ORF1b and $\mathrm{N}$, and pCMV-MCS-upE containing upE, are presented in Figure 1(a). After expression, the pellets obtained were visualized by transmission electron microscopy to confirm that VLPs were produced. As shown in Figure 1(b), the average particle size was $100 \mathrm{nM}$, similar to that reported previously [14]. The concentrations of MERS-CoV upEVLPs determined by the ddPCR assay were $4.701 \pm 1.102 \times 10^{8}$ copies $/ \mathrm{mL}$ while MERS1bNVLPs were $2.730 \pm 1.483 \times$ $10^{8}$ copies/mL. By comparing the copy numbers obtained without the reverse transcription (Table 2), the plasmid DNA pollution in the VLP solutions was low, from $1 \%$ to $2.76 \%$ of the total copy numbers obtained after the reverse transcription. 


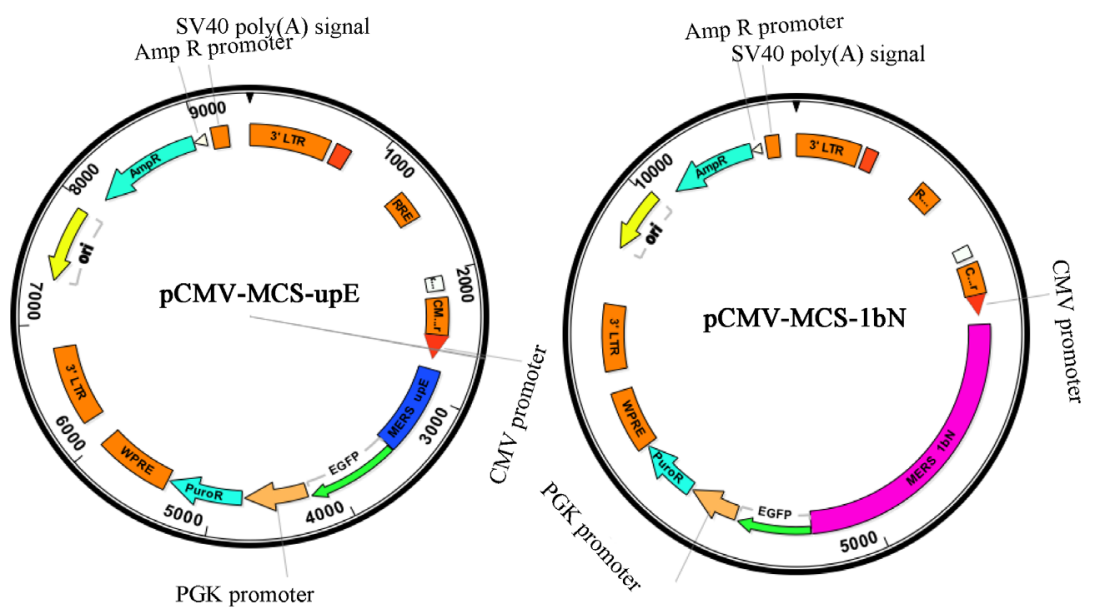

(a)

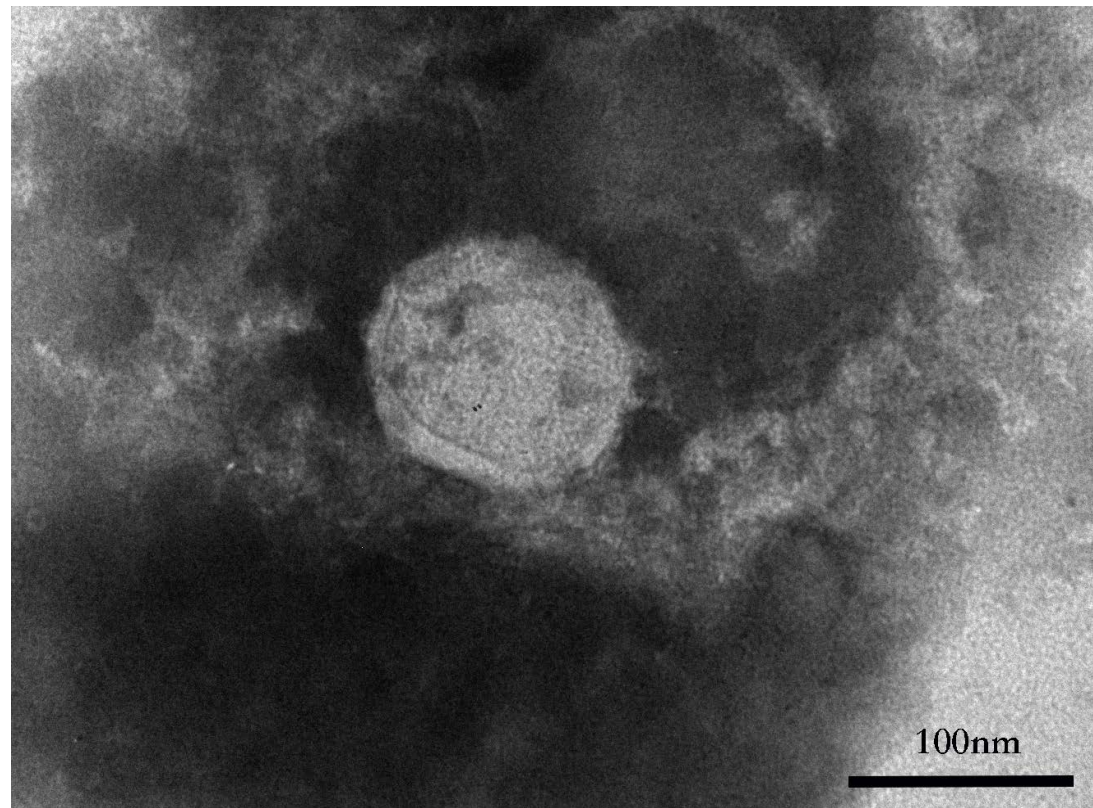

(b)

Figure 1. (a) MERS-CoV sequences of upE (Dark blue) and $1 \mathrm{bN}$ (Pink) were subcloned into the lentiviral vectors between the restriction sites $E c o R 1$ and $B a m H 1$, respectively. The main elements of the lentiviral vector used for the production of viral RNA and incorporation within HIV-like particle are indicated; (b) A representative image of the MERSVLPs analyzed by negative staining transmission electron microscopy. The magnification times is $10,000 \times$, the average particle size is $100 \mathrm{~nm}$ (average of 6 fields).

Table 2. DNA contaminations in MERS-CoV VLPs determined by ddPCR.

\begin{tabular}{cccc}
\hline Samples & $\begin{array}{c}\text { Reverse transcription } \\
\text { procedure }\end{array}$ & $\begin{array}{c}\text { Concentration of } \\
\text { templates }(\text { copies/mL) }\end{array}$ & DNA contamination (\%) \\
\hline MERS-upE & + & $1.27 \times 10^{10}$ & 2.76 \\
MERS-upE & - & $3.6 \times 10^{8}$ & \\
MERS-1bN & + & $1.01 \times 10^{10}$ & 1.02 \\
MERS-1bN & - & $1.05 \times 10^{8}$ & \\
\hline
\end{tabular}




\subsection{Evaluation of MERS-CoV VLPs as the Positive Control Material in NAT}

By reconstituting the lyophilized MERSVLPs with PBS buffer, the resulted solution could be used as the positive control in the NAT assay kits. As shown in Figure 2, good linearity $\left(\mathrm{R}^{2}=0.99537\right.$ for MERS1bNVLPs, and $\mathrm{R}^{2}=0.99981$ for MERSupEVLPs) was obtained using the commercial qPCR kit to test serial dilutions of the VLP solutions ranging from $10^{2}-10^{7}$ copies $/ \mathrm{mL}$. The lyophilized powders of the MERS-CoV VLPs were quite stable and no significant differences in the form of $\Delta \log$ (copies $/ \mathrm{mL}$ ) were found using the ddPCR after storing at $-20^{\circ} \mathrm{C}, 4^{\circ} \mathrm{C}$ and $37^{\circ} \mathrm{C}$ for 7 days (Table 3 ).

\subsection{Evaluation of MERS-CoV VLPs as the Reference Material in EQA}

In EQA, a sample panel with different concentrations is needed. Therefore, we tested the homogeneity of the MERS-CoV VLP solutions reconstituted with the virus preservation solution. As shown in Figure 3, repeated testing of the solutions showed very low variations, with $\mathrm{CV}$ less than $0.6 \%$. Further testing the

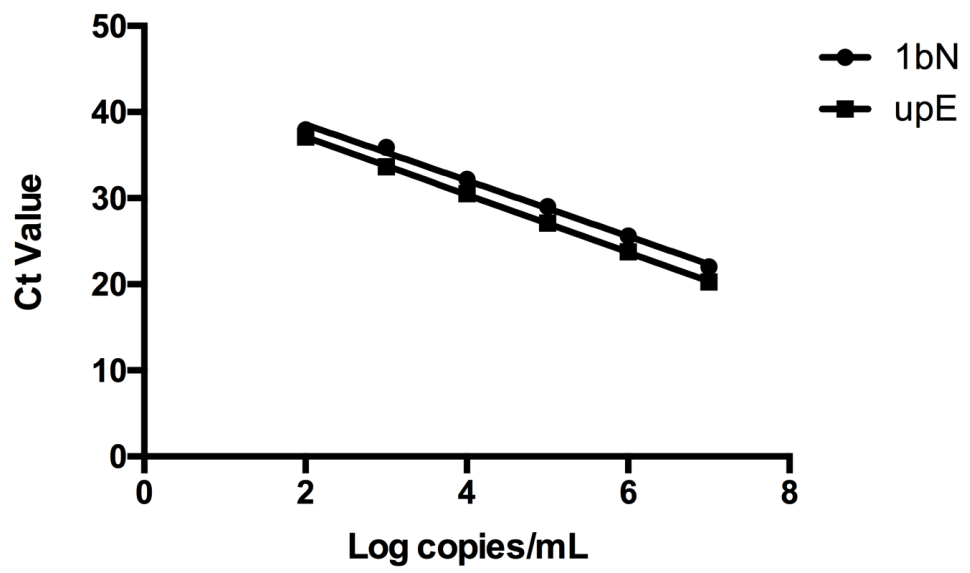

Figure 2. Standard curves for qPCR determination of upE of MERS-CoVVLPupE (square) and $1 b N$ of MERS-CoVVLP1bN (dot) after RNA extraction by the commercial kit. The extracted RNAs were tenfold diluted serially from $10^{7}$ to $10^{2}$ copies/uL. The slope of the MERS-CoVVLPupE standard curve is $-3.350\left(\mathrm{R}^{2}=0.99981\right)$. The slope of the MERS-CoVVLP1bN standard curve is $-3.244\left(\mathrm{R}^{2}=0.99537\right)$. The linear equation is below: $\mathrm{CT}=-\mathrm{klg} \mathrm{X} 0+\mathrm{b}$.

Table 3. Stability of the lyophilized MERS-CoVVLPs powders after 7 days of storage at different temperatures.

\begin{tabular}{cccc}
\hline & $-20^{\circ} \mathrm{C}$ & $4^{\circ} \mathrm{C}$ & $37^{\circ} \mathrm{C}$ \\
\hline MERS-upE & 0 & -0.0494 & -0.6204 \\
MERS-1bN & 0 & -0.0679 & -0.6919
\end{tabular}

Samples stored at different temperatures are assessed by measuring the copy numbers of upE and ORF1busing the ddPCR. $\triangle \log ($ copies $/ \mathrm{mL})$ are calculated by $\log \left({ }^{\circ} \mathrm{T}\right)-\log \left(-20^{\circ} \mathrm{C}\right)$. The data in the table represent the average of two repeats. 


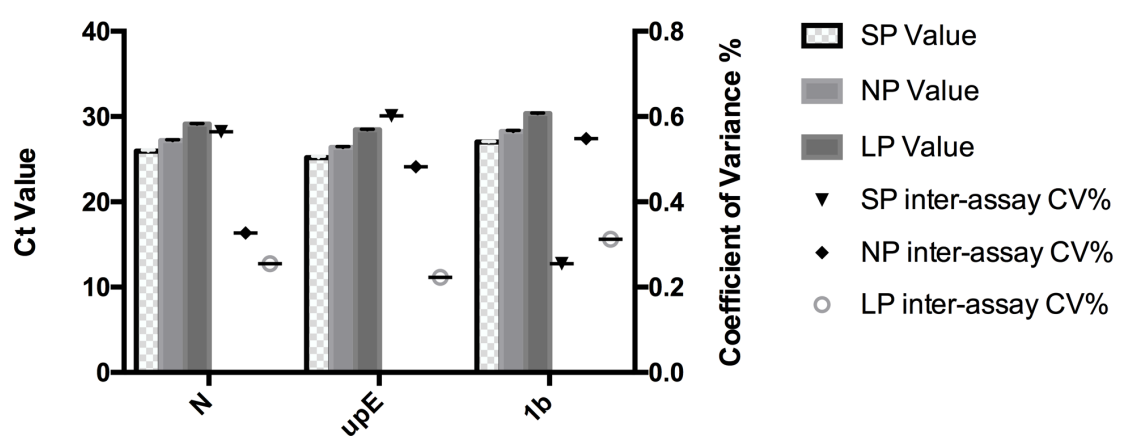

Figure 3. Homogeneity of the MERSVLPs solution prepared by reconstituting the lyophilized powder with the virus protection solution. Sample SP: $1 \times 10^{6}$ copies $/ \mathrm{mL}$, Sample NP: $5 \times 10^{5}$ copies $/ \mathrm{mL}$, and Sample LP: $1 \times 10^{5}$ copies $/ \mathrm{mL}$. Histogram indicates the average $\mathrm{Ct}$ values of each sample. Dots show variation of CV of the qPCR assay in repeated tests of the solutions.

stability of the sample panel under different storage temperatures showed that the MERS-CoV VLP solutions were stable after storing at $-70^{\circ} \mathrm{C},-20^{\circ} \mathrm{C}$ and $4^{\circ} \mathrm{C}$ for at least one week (Figure 4). But the Ct values of the MERS-CoV VLPs solutions stored at $37^{\circ} \mathrm{C}$ went up gradually over time, showing that the MERS VLPs were degraded gradually at $37^{\circ} \mathrm{C}$. These results showed that the sample panel solutions prepared from the lyophilized MERS-CoV VLP powders should be stored under cooling conditions.

\subsection{EQA Results of 49 Laboratories}

As summarized in Table 4, all the 49 laboratories reported negative and positive results $100 \%$ correct after testing the sample panel consisting of four different concentrations of the MERS VLPs (negative control, LP, NP and SP) although different extraction methods and detection kits were used. However, the Ct values between different labs were varied significantly (Figure 5) when testing the VLP solutions at the same concentration level, the $\mathrm{P}$ value is $>0.05$.

\section{Discussion}

Because RT-PCR detection of MERS-CoV involves many steps, from RNA extraction, reverse transcription to PCR, any of the steps would cause the detection failure. Therefore, it is important to have a positive control material which could be used to monitor the whole process of the RT-PCR detection.

In this study, we have tried to use the lentivirus system to pack extraneous MERS-CoV RNA fragments inside the virus-like particles. The first advantage of this approach is that the final VLPs are safe for in vitro uses because of lacking of self-reproduction ability. Secondly, the lyophilized powders of the MERS-CoVVLPs are stable during storage at $4^{\circ} \mathrm{C}$, even at $37^{\circ} \mathrm{C}$ over a week. These results imply that the lyophilized powders are suitable for use as the positive control material in PCR detection kits. Finally, although the lentivirus system has relatively larger packing capacity ( $4 \mathrm{k}$ maximum) than that of MS2 bacteriophages, 

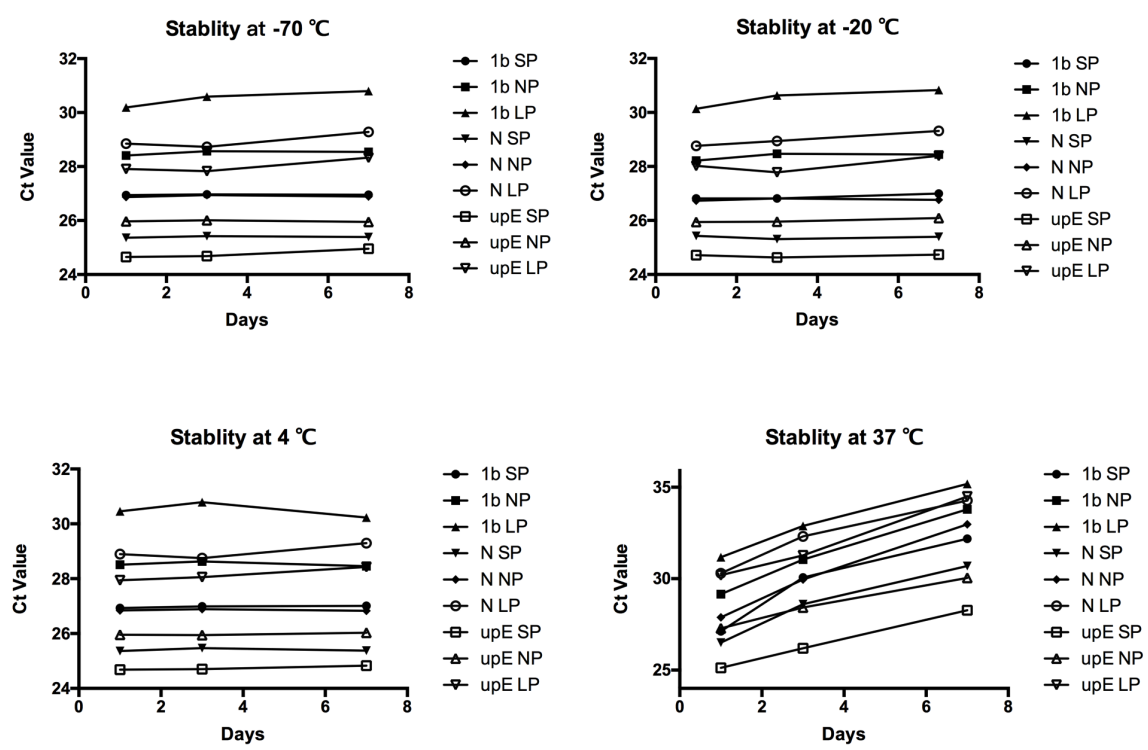

Figure 4. Stability of the VLPs solutions after one week storage at different temperatures from 1 day to 7 days. Samples are assessed by quantitative RT-PCR detection of ORF1 $b$ and upE.

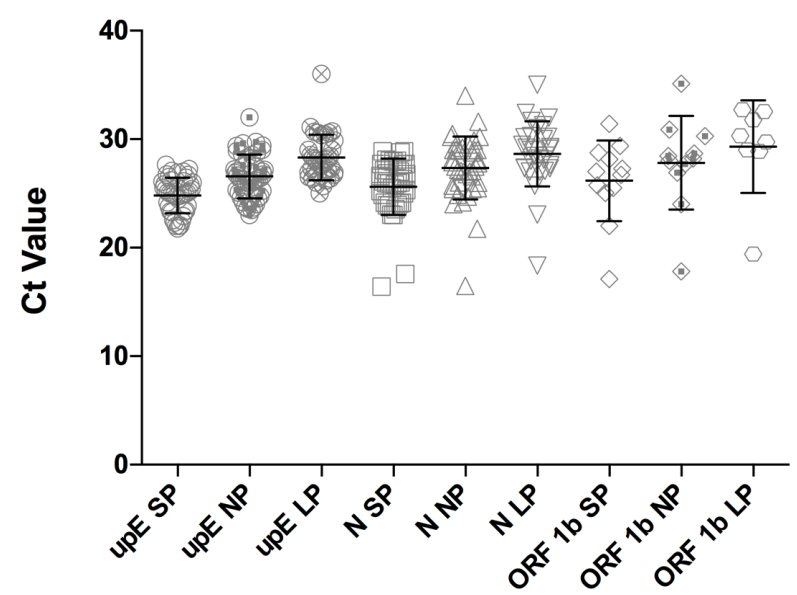

Figure 5. Scatter diagram showing the Ct values (y-axis) and samples ( $\mathrm{x}$-axis) obtained by all the labs participating the EQA.

which could be exploited to load more genes of MERS-CoV, the whole genome of the MERS-CoV is still too big to be encapsulated into one HIV-1 VLP. However, since commercial diagnosis kits normally target at only 1 or 2 conserved gene fragments of MERS-CoV, the load volume of the lentivirus packing system should have no problem at all for constructing positive controls in PCR kits.

Besides using as the positive control material in PCR detection kits, the constructed MERS-CoV VLPs were also used to prepare a sample panel for EQA. The VLP solutions prepared by reconstitution with the virus preservation solution were found homogenous and stable at $4^{\circ} \mathrm{C}$ over a week. The initial EQA results 
Table 4. Information and results of the 49 laboratories participating the EQA for MERS-CoV qPCR detection.

\begin{tabular}{|c|c|c|c|c|}
\hline Lab No. & Extraction kit ${ }^{\mathrm{a}}$ & Extraction method & Real time RT-PCR assay ${ }^{\mathrm{b}}$ & Agreement (\%) \\
\hline 1 & RNAeasy & Magnetic bead & A & 100 \\
\hline 2 & KINGFISHER & Magnetic bead & A & 100 \\
\hline 3 & $-*$ & - & $\mathrm{A}$ & 100 \\
\hline 4 & - & - & A & 100 \\
\hline 5 & Daan gene & Magnetic bead & $\mathrm{C}$ & 100 \\
\hline 6 & - & - & $\mathrm{A}$ & 100 \\
\hline 7 & - & - & A & 100 \\
\hline 8 & QIAamp & Spin column & A & 100 \\
\hline 9 & - & - & $\mathrm{F}$ & 100 \\
\hline 10 & QIAamp & Spin column & A & 100 \\
\hline 11 & PROMEGA & Spin column & A & 100 \\
\hline 12 & - & - & A & 100 \\
\hline 13 & QIAamp & Spin column & A & 100 \\
\hline 14 & - & - & A & 100 \\
\hline 15 & QIAamp & Spin column & B & 100 \\
\hline 16 & QIAamp & Spin column & A & 100 \\
\hline 17 & QIAamp & Spin column & A & 100 \\
\hline 18 & QIAEZ1 & Magnetic bead & $\mathrm{D}$ & 100 \\
\hline 19 & - & - & A & 100 \\
\hline 20 & QIAamp & Spin column & A & 100 \\
\hline 21 & ZJ Bio-Tech & Magnetic bead & A & 100 \\
\hline 22 & QIAamp & Spin column & A & 100 \\
\hline 23 & QIAamp & Spin column & A & 100 \\
\hline 24 & - & - & A & 100 \\
\hline 25 & - & - & A & 100 \\
\hline 26 & - & - & A & 100 \\
\hline 27 & QIAamp & Spin column & $\mathrm{A}$ & 100 \\
\hline 28 & QIAamp & Spin column & B & 100 \\
\hline 29 & - & - & A & 100 \\
\hline 30 & - & - & A & 100 \\
\hline 31 & High viral RNA & Magnetic bead & A & 100 \\
\hline 32 & QIAamp & Spin column & B & 100 \\
\hline 33 & ZJ Bio-Tech & Magnetic bead & A & 100 \\
\hline 34 & - & - & A & 100 \\
\hline 35 & - & - & $\mathrm{A}$ & 100 \\
\hline
\end{tabular}


Continued

\begin{tabular}{lcccc}
\hline 36 & - & - & A & 100 \\
37 & - & - & A & 100 \\
38 & QIAEZ1 & Magnetic bead & E & 100 \\
39 & - & - & A & 100 \\
40 & QIAamp & Spin column & A & 100 \\
41 & - & - & A & 100 \\
42 & Tianlong & Magnetic bead & G & 100 \\
43 & QIAamp & Spin column & H & 100 \\
44 & - & - & B & 100 \\
45 & - & - & A & 100 \\
46 & QIAEZ1 & Magnetic bead & A & 100 \\
47 & - & - & A & 100 \\
48 & MagMax & Magnetic bead & A & 100 \\
49 & - & - & A & 100 \\
\hline
\end{tabular}

a: QIAamp, QIAamp viral RNA minikit (Qiagen); KINGFISHER, KingFisher Pure RNA Tissue Kit (ThermoScientic); PROMEGA, SV Total RNA Isolation System (Promega); Daan gene, Daan gene viral RNA kit; RNeasy, RNeasy minikit (Qiagen); ZJ Bio-Tech, ZJ Bio-Tech viral RNA kit; QIAEZ1, QiagenEZ1 virus mini kit (Qiagen); High viral RNA, High Pure Viral RNA Kit (Roche); Tianlong, Tianlong viral RNA kit; MagMax, ABI Life Technologies AM 1836 MagMAX-96 ViralRNA Isolation Kit. ${ }^{\text {b: }}$ A: ZJ Bio-Tech (ZJ Bio-Tech Co., Ltd., Shanghai, China); B: BioPerfectus (BioPerfectus Technologies, Jiangsu, China); C: Daan Gene (Daan Gene Co., Ltd., Guangzhou, China); D: primers offered by USA CDC; E: primers offered by China CDC; F: Mokobio (Mokobio Biotechnology Co., ltd. Nanjing, China); G: TianLong (TianLong Bio-Tech Co., Ltd., Suzhou, China); H: HuiruiBio (Shanghai Huirui Biotechnology Co., Ltd., Shanghai, China); -: not provided.

from 49 labs demonstrated that the constructed VLPs could be well used as the reference material for EQA. At the same time, the different $\mathrm{Ct}$ values reported with different labs for the same sample would hint that there need to standardize the RNA extraction method and/or the PCR detection conditions between the labs.

\section{Conclusion}

In conclusion, we have successfully constructed two MERS-CoV VLPs and demonstrated that they are stable and safe to be used as the positive control in PCR detection kits as well as the reference material in EQA.

\section{Acknowledgements}

This work was supported by Scientific Research Grant No. 2016IK176 and No. T0217-NBCIQ-0016 from General Administration of Quality Supervision, Inspection and Quarantine of the People's Republic of China.

\section{Competition of Interests}

None declared. 


\section{References}

[1] Shirato, K., Yano, T., Senba, S., Akachi, S., Kobayashi, T., Nishinaka, T., et al. (2014) Detection of Middle East Respiratory Syndrome Coronavirus Using Reverse Transcription Loop-Mediated Isothermal Amplification (RT-LAMP). Virology Journal, 11, 139. https://doi.org/10.1186/1743-422X-11-139

[2] Mackay, I.M. and Arden, K.E. (2015) MERS Coronavirus: Diagnostics, Epidemiology and Transmission. Virology Journal, 12, 222.

[3] Corman, V.M., Eckerle, I., Bleicker, T., Zaki, A., Landt, O., Eschbach-Bludau, M., et al. (2012) Detection of a Novel Human Coronavirus by Real-Time Reverse-Transcription Polymerase Chain Reaction. Eurosurveillance, 17, 3-8. https://doi.org/10.2807/ese.17.39.20285-en

[4] Pereyaslov, D., Rosin, P., Palm, D., Zeller, H., Gross, D., Brown, C.S., et al. (2014) Laboratory Capability and Surveillance Testing for Middle East Respiratory Syndrome Coronavirus Infection in the WHO European Region, June 2013. Eurosurveillance, 19, 26-34. https://doi.org/10.2807/1560-7917.ES2014.19.40.20923

[5] Corman, V.M., Muller, M.A., Costabel, U., Timm, J., Binger, T., Meyer, B., et al. (2012) Assays for Laboratory Confirmation of Novel Human Coronavirus (hCoV-EMC) Infections. Eurosurveillance, 17, 2-10. https://doi.org/10.2807/ese.17.49.20334-en

[6] Blaise-Boisseau, S., Hennechart-Collette, C., Guillier, L. and Perelle, S. (2010) Duplex Real-Time qRT-PCR for the Detection of Hepatitis A Virus in Water and Raspberries Using the MS2 Bacteriophage as a Process Control. Journal of Virological Methods, 166, 48-53. https://doi.org/10.1016/j.jviromet.2010.02.017

[7] Dreier, J., Stormer, M. and Kleesiek, K. (2005) Use of Bacteriophage MS2 as an Internal Control in Viral Reverse Transcription-PCR Assays. Journal of Clinical Microbiology, 43, 4551-4557. https://doi.org/10.1128/JCM.43.9.4551-4557.2005

[8] Rolfe, K.J., Parmar, S., Mururi, D., Wreghitt, T.G., Jalal, H., Zhang, H., et al. (2007) An Internally Controlled, One-Step, Real-Time RT-PCR Assay for Norovirus Detection and Genogrouping. Journal of Clinical Virology, 39, 318-321. https://doi.org/10.1016/j.jcv.2007.05.005

[9] Trombley, A.R., Wachter, L., Garrison, J., Buckley-Beason, V.A., Jahrling, J., Hensley, L.E., et al. (2010) Short Report: Comprehensive Panel of Real-Time TaqMan (TM) Polymerase Chain Reaction Assays for Detection and Absolute Quantification of Filoviruses, Arenaviruses, and New World Hantaviruses. American Journal of Tropical Medicine and Hygiene, 82, 954-960. https://doi.org/10.4269/ajtmh.2010.09-0636

[10] Towner, J.S., Sealy, T.K., Ksiazek, T.G. and Nichol, S.T. (2007) High-Throughput Molecular Detection of Hemorrhagic Fever Virus Threats with Applications for Outbreak Settings. The Journal of Infectious Diseases, 196, S205-S212. https://doi.org/10.1086/520601

[11] Reusken, C., Niedrig, M., Pas, S., Anda, P., Baize, S., Charrel, R., et al. (2015) Identification of Essential Outstanding Questions for an Adequate European Laboratory Response to Ebolavirus Zaire West Africa 2014. Journal of Clinical Virology, 62, 124-134. https://doi.org/10.1016/j.jcv.2014.11.007

[12] Wang, G.J., Sun, Y., Zhang, K., Jia, T.T., Hao, M.J., Zhang, D., et al. (2015) External Quality Assessment of Molecular Detection of Ebola Virus in China. PLoS ONE, 10, e0151695. https://doi.org/10.1371/journal.pone.0132659

[13] Shahkarami, M., Yen, C., Glaser, C., Xia, D.X., Watt, J. and Wadford, D.A. (2015) 
Laboratory Testing for Middle East Respiratory Syndrome Coronavirus, California, USA, 2013-2014. Emerging Infectious Diseases, 21, 1664-1666.

https://doi.org/10.3201/eid2109.150476

[14] Papanikolaou, E., Kontostathi, G., Drakopoulou, E., Georgomanoli, M., Stamateris, E., Vougas, K., et al. (2013) Characterization and Comparative Performance of Lentiviral Vector Preparations Concentrated by Either One-Step Ultrafiltration or Ultracentrifugation. Virus Research, 175, 1-11.

https://doi.org/10.1016/j.virusres.2013.03.015 